\title{
Integrated Cancer Repository for Cancer Research
}

National Cancer Institute

\section{Source}

National Cancer Institute. integrated Cancer Repository for Cancer Research. NCI

Thesaurus. Code C122420.

A system for the standardized collection, mining and sharing of comprehensive cancerrelated data. It consists of registry databases and human biological material. Currently, there are seven registries/tissue banks developed under the umbrella of iCaRe2: the Pancreatic Cancer Collaborative Registry, Thyroid Cancer and Tumor Collaborative Registry, GenitoUrinary Cancer Collaborative Registry, Thoracic Oncology Collaborative Registry, Gastrointestinal and Abdominal Cancer Collaborative Registry, Head and Neck Cancer Collaborative Registry, and the Central Nervous System Tumor Collaborative Registry for collaborative multi-center research. 